\title{
Über Phasengleichgewichte im System: Wismut und Schwefel.
}

Von

\author{
A. H. W. Aten.
}

Mit 6 Figuren im Text und 1 Tafel.

Bis jetzt sind umfassendere Phasengleichgewichte, worin Ver. bindungen zweier Komponenten auftreten, nur an wenig beständigen Molekularverbindungen, Hydraten, Verbindungen von Metallen untereinander usw. studiert worden; von den stabileren chemischen Verbindungen, wie Salzen, Oxyden, Sulfiden, ist in dieser Hinsicht wenig bekannt, zum Teil wegen der experimentellen Schwierigkeiten, welche ein solches Studium in vielen Fällen mit sich bringt.

Sebr interessant ist daher die Arbeit Ptlabons über die Schmelzerscheinungen von Mischungen von Wismut oder Antimon mit Schwefel oder Selen, ${ }^{1}$ wenn er auch die experimentellen Ergebnisse nach meiner Meinung nicht richtig deutet. Ich möchte deshalb seine Resultate, speziell hinsichtlich des Systems $B i+S$, auf eine andere Weise betrachten und habe die Schmelzlinie von $\mathrm{Bi}+\mathrm{S}$ noch einmal bestimmt. Die Schwefelwismut-Mischungen wurden ebenso wie bei Pex_abon, in Glasröhren geschmolzen, ein Thermoelement, das mit einem Galvanometer verbunden war, wurde eingeführt, und der Anfangs- und Enderstarrungspunkt aus dem Verlauf der Abkühlungskurve gefunden. Wenn dreimal derselbe Punkt gefunden war, wurde die Mischung analysiert, indem der Schwefel als $\mathrm{BaSO}_{4}$ bestimmt wurde. Die gefundenen Erstarrungspunkte sind in folgender Tabelle zusammengestellt, in Fig. 1 ist die Erstarrungskurve gezeichnet worden.

1 Journ. chim. phys. 2 (1904), 320. 


\section{- $387-$}

Zusumm.

in Atomproz. $\mathrm{S}$

Anfangs-

End-

Erstarrungspunkt in ${ }^{\circ}$

0.0

277

0.9

291

4.2

17.2

28.8

40.7

46.9

277

318

272

452

263

535

267

602

270

52.4

760

$-$

$$
80
$$

$800^{\circ}$

$700^{\circ}-\frac{1}{2}$

$$
6
$$

60

$$
50
$$

600

550

580
450

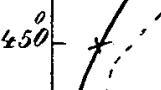

400

400

35

300

250

$B i$

$x \quad x \quad x$

Zusamm. in Atopripicz. S

Fig. 1.

Die beiden Erstarrungspunkte kamen gewöhnlich sehr deutlich zum Vorschein, nur an den beiden letzteren Mischungen war der Enderstarrungspunkt nicht mehr zu beobachten. 
Die gefundene Schmelzkurve stimmt im grofsen ganzen mit derjenigen von Pruabon, d.i. mit der punktierten Linie in Fig. 1 überein; die Kurve verläuft an der Wismutachse sehr steil, zeigt dann einen flacheren Teil und steigt dann wieder etwas stärker. Letzteres ist bei Pelabon nicht der Fall, er findet im Gegenteil, dal's von 50 Atomproz. S ab die Kurve weniger steil geht. Dafs die Schmelz. kurve nicht weiter als bis etwa 55 Atomproz. Schwefel verfolgt werden kann, werden wir später betrachten.

Die Frage, was aus dem Verlaufe der Erstarrungskurve zu schliefsen ist, beantwortet Pélabon in dem Sinne, dafs durch den Knick in seiner Kurve bei 50 Atomproz. $\mathrm{S}$ die Existenz der Verbindung BiS bewiesen sei. Er sagt: „En resumé les sulfures (et seléniures) de bismuth répondant aux formules BiS (et BiSe) sont nettement mis en évidence par les courbes de fusibilite des mélanges, que forment ces corps soit avec un excès de métal, soit avec un excès de métalloïde."

Ich kann dieser Meinnng nicht beistimmen. Betrachten wir zuerst die Kurve Pélabons. Der Punkt bei $50 \%$ kann kein Schmelzpunkt des BiS darstellen, denn dann mülste die Kurve von dortab wieder absteigen. Ebensowenig kann er ein Übergangspunkt sein, wobei die feste Phase $\mathrm{BiS}$ in eine schwefelreichere z. B. $\mathrm{Bi}_{2} \mathrm{~S}_{3}$ übergeht, denn in diesem Falle würde die Schmelzkurve für $\mathrm{Bi}_{2} \mathrm{~S}_{3}$ steiler laufen als die Kurve für BiS, was bei PtLabon nicht der Fall ist. 1

In meiner Kurve ist dagegen bei $50 \%$ kein besonderer Punkt anwesend. Es konnte jedoch noch angenommen werden, dafs bei einer zwischen $\mathrm{BiS}$ und $\mathrm{Bi}_{2} \mathrm{~S}_{3}$ gelegenen Zusammensetzung ein eutektischer Punkt anwesend wäre, welcher sehr nahe an der $\mathrm{Zu}$ sammensetzung BiS und dadurch so wenig unterhalb dessen Schmelzpunktes gelegen wäre, dals er von mir bei der Bestimmung der Anfangspunkte der Erstarrung übersehen wäre.

Es mulste dann in der Abkühlungskurve der Gemische mit mehr als 50 Atomproz. S ein Enderstarrungspunkt, wenig unterhalb des Schmelzpunktes von BiS gefunden sein. Hiervon ist jedoch gar nichts bemerkt, wie deutlich durch die in Fig. 5 dargestellte Abkühlungskurve der Mischung mit 52.4 $\mathrm{S}$ gezeigt wird.

Bei $760^{\circ}$ ist ein Knick anwesend, der dem Anfangspunkt der

${ }^{1}$ Ebenso ist die Richtung der Kurven in Péánons Knickpunkte bei $450^{\circ}$ fehlerhaft. Ich habe auch dort eine kontinuierliche Kurve gefunden. 


\section{$-\quad 389$}

Erstarrung entspricht, sonst zeigt die Kurve einen ganz regelmärsigen Verlauf.

Ich schliefse daher aus meinen Erstarrungsversuchen, dafs die ganze Kurve von 0.1-52.4 Atomproz. S die Schmelzkurve eines

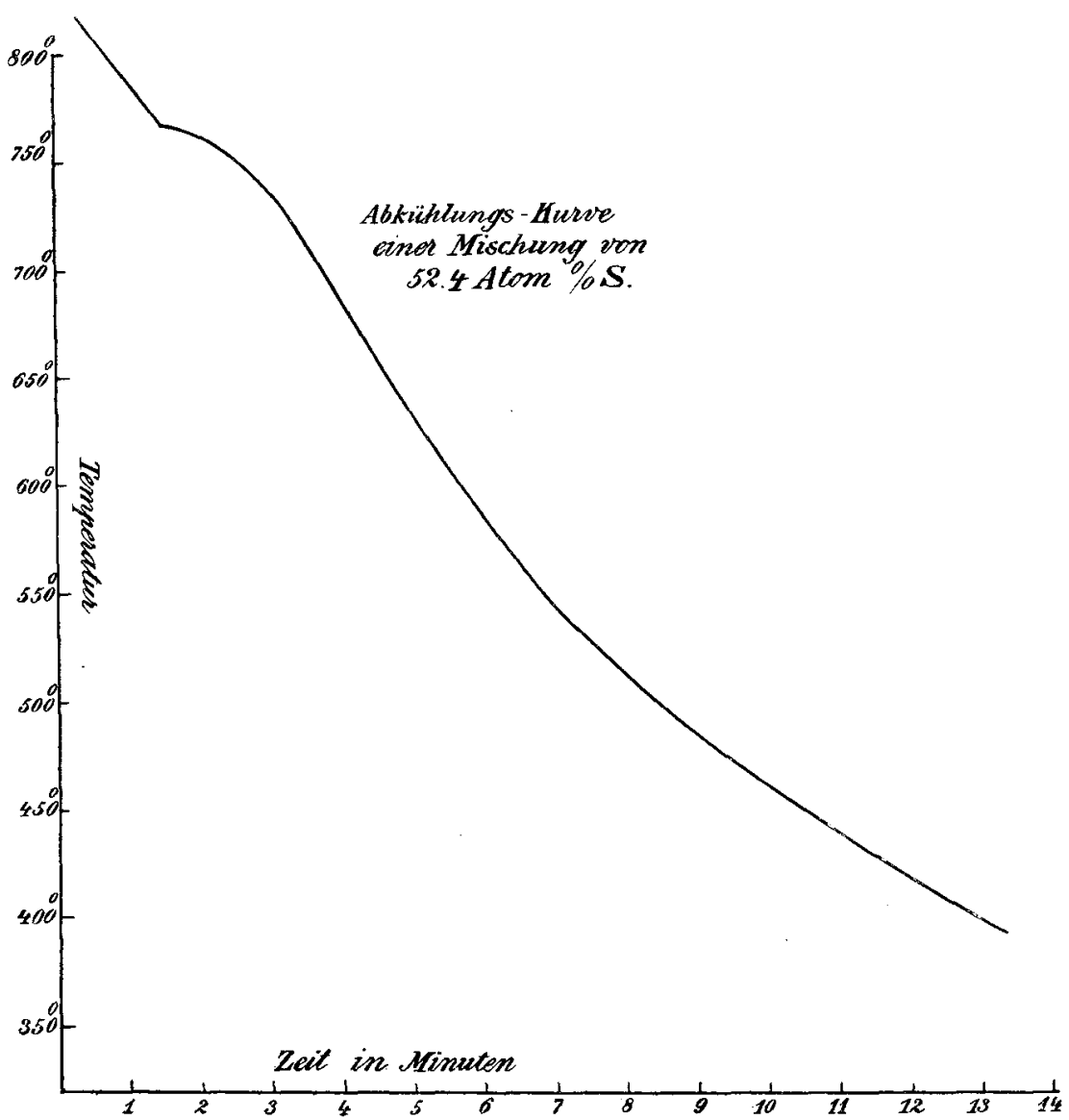

Fig. 2.

und desselben Sulfids ist mit mehr Schwefel als BiS, vermutlich $\mathrm{Bi}_{2} \mathrm{~S}_{3}$. $\mathrm{Zu}$ demselben Resultat führt die mikroskopische Untersuchung der polierten und geätzten Schwefelwismutmischungen.

$\mathrm{Zu}$ diesem $\mathrm{Z}$ weck wurden die Mischungen, welche zur Bestimmung der Erstarrungspunkte gedient hatten, durchgesägt, die Oberfläche auf Schmirgelpapier geschliffen und schliefslich mit Chromoxyd poliert. Schon durch blofses Schleifen trat die Struktur 
an den Tag, deutlicher wurde dieselbe durch Ätzen mit Salzsäure und Ferrichlorid.

Die Bilder dieser Schliffe sind in den Fig. 3-8 reproduziert worden.

Die Bilder zeigen ziemlich scharf begrenzte Kristalle, deren Menge mit dem Schwefelgehalte zunimmt, die Kristalle stellen das Wismutsulfid dar, der meistens dunkel gefärbte Grund wird das Eutektikum von Wismut und Wismutsulfid sein, das fast gänzlich aus ersterem besteht. Die Mischungen von 46.9 und 52.4 Atom. proz. $\mathbf{S}$ geben ein vollkommen vergleichbares Bild, nur enthält die Mischung von 52.4 Atomproz. mehr Kristalle von Wismutsulfid. Dies könnte nicht der Fall sein, wenn sich in der Mischung von 46.9 Atomproz. S, BiS neben Bi befand, in der Mischung von 52.4 Atomproz. $\mathrm{S}$ aber BiS neben $\mathrm{Bi}_{2} \mathrm{~S}_{3}$; denn dann berechnet sich für 52.4 Atomproz. $\mathrm{S}$ eine geringere Menge Kristalle von BiS. Das mikrografische Studium gibt also auch keinen Grund zur Annahme, dafs BiS als feste Phase auftritt.

Die nächstliegende Annahme, dafs zur Kurve, die bis zu $52 \% \mathrm{~S}$ studiert wurde, $\mathrm{Bi}_{2} \mathrm{~S}_{3}$ als feste Phase gehört, stimmt dagegen ganz gut mit der Masse des Sulfids in den unterschiedenen Schliffen überein.

Sie konnte durch die Lage der eutektischen Linie bei $270^{\circ}$ gestützt werden, wenn diese auch noch zwischen 50 und 60 Atomproz. S gefunden wäre. Dals das nicht der Fall war, braucht jedoch nicht als streitig angemerkt zu werden, da der Gehalt am Eutektikum in solchen Gemischen darum äufserst gering ist, weil der eutektische Punkt so nahe an der Wismutachse liegt.

Es ist schade, dals die Schmelzkurve nicht über 55 Atomproz. $\mathrm{S}$ hat fortgesetzt werden können, denn die Fortsetzung bis zu $60 \%$ und darüber würde vollkommene Sicherheit gegeben haben.

Die Unmöglichkeit der Fortsetzung hat einfach seinen Grund darin, dafs die Mischung mit $55 \% \mathrm{~S}$ beim Erhitzen bis zu ihrem Schmelzpunkte zu sieden anfängt, die Schmelzkurve wäre also nur bei höheren Drucken weiter zu verfolgen, was aber im Glasröhrchen unmöglich ist, weil das Glas bei diesen Temperaturen schon ziemlich weich ist.

Es kommt also darauf hinaus, dals die Siedekurve die Schmelzkurve schneidet, wodurch ein Teil beider Kurven fortfällt.

Bei einer Lage der Schmelz- und Siedepunkte wie hier, (der Siedepunkt der Verbindung liegt zwischen denjenigen der Komponenten, der Schmelzpunkt der Verbindung dagegen höher als die- 
jenigen der Komponenten) wird der Schnitt auf zwei verschiedene Arten stattfinden können, wie durch die Fig. $9 \mathrm{a}$ und $9 \mathrm{~b}$ angezeigt werden kann, wenn nämlich die Verbindung bei ihrem Siedepunkte nicht dissoziiert ist. Diese Figuren stellen nur eine Verknüpfung zweier Fälle dar, welche bei einem binären Systeme ohne Verbindung

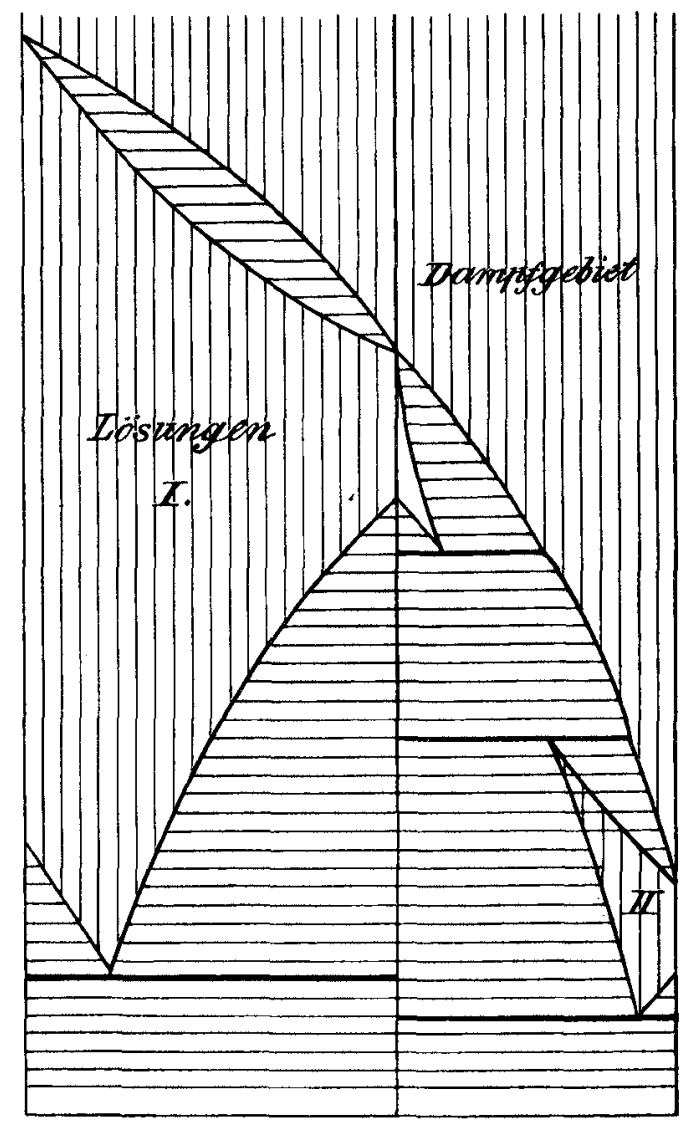

Fig. 9 a.

stattfinden können. (Man vergleiche die Ableitung bei BAKHUIS Roozeвoom, Heterogene Gleichgewichte II, 1, S. 333 ff.) Fig. 9a gilt für den Fall, dals der Tripelpunktsdruck der Verbindung niedriger, Fig. $9 \mathrm{~b}$ wenn derselbe höher liegt als eine Atmosphäre, wenn also die Verbindung bei diesem Drucke sublimiert.

Nehmen wir jetzt an, dals die Verbindung bei ihrem Siedepunkte und auch bei ihrem Schmelzpunkte dissoziiert ist, so wird 
hierdurch eine Formänderung der Siede- und Schmelzkurven bedingt. Die Siedekurven gehen auseinander, der Schmelzpunkt der Verbindung sinkt bei zunehmender Dissoziation und die Schmelzkurve wird abgeflacht.

Bei geringerer Dissoziation kann die Änderung der Kurven in Fig. $9 \mathrm{a}$ und $9 \mathrm{~b}$ so gering sein, dafs die allgemeine Verknüpfung

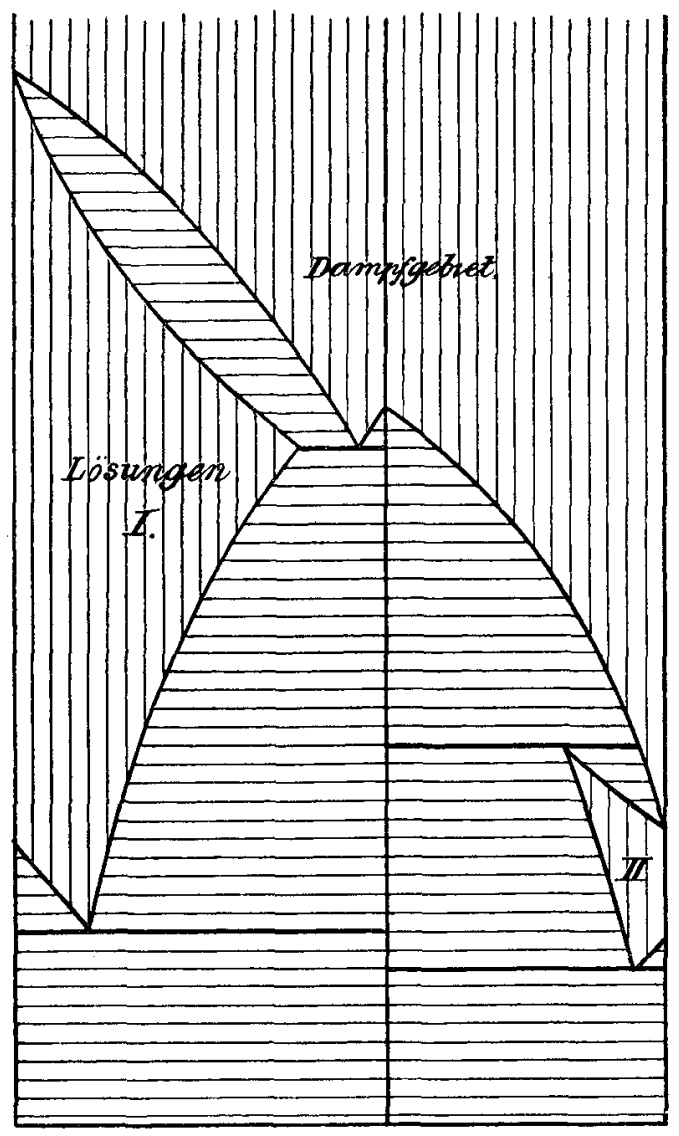

Fig. $9 \mathrm{~b}$.

der Gebiete dadurch noch nicht beeinflurst wird, so wenn aus Fig. 9 a Fig. 9c entsteht. Bei stärkerer Dissoziation kann jedoch auch die Verknüpfung der Gebiete geändert werden, und kann Fig. $9 \mathrm{~d}$ sowohl aus $9 \mathrm{a}$ als $9 \mathrm{~b}$ entstehen, Fig. 9d ist jetzt geeignet, die beim System $\mathrm{Bi}+\mathrm{S}$ angetroffenen Verhältnisse klar zu legen. Es ist dabei an- 
genommen, dafs $\mathrm{Bi}_{2} \mathrm{~S}_{3}$ die sich bildende Verbindung ist. Betrachten wir die Figur etwas näher.

$A$ und $B$ sind die Siede- und Schmelzpunkte von Wismut, $C$ und $D$ von Schwefel. $A H, K C, A I, L C$ sind die realisierbaren

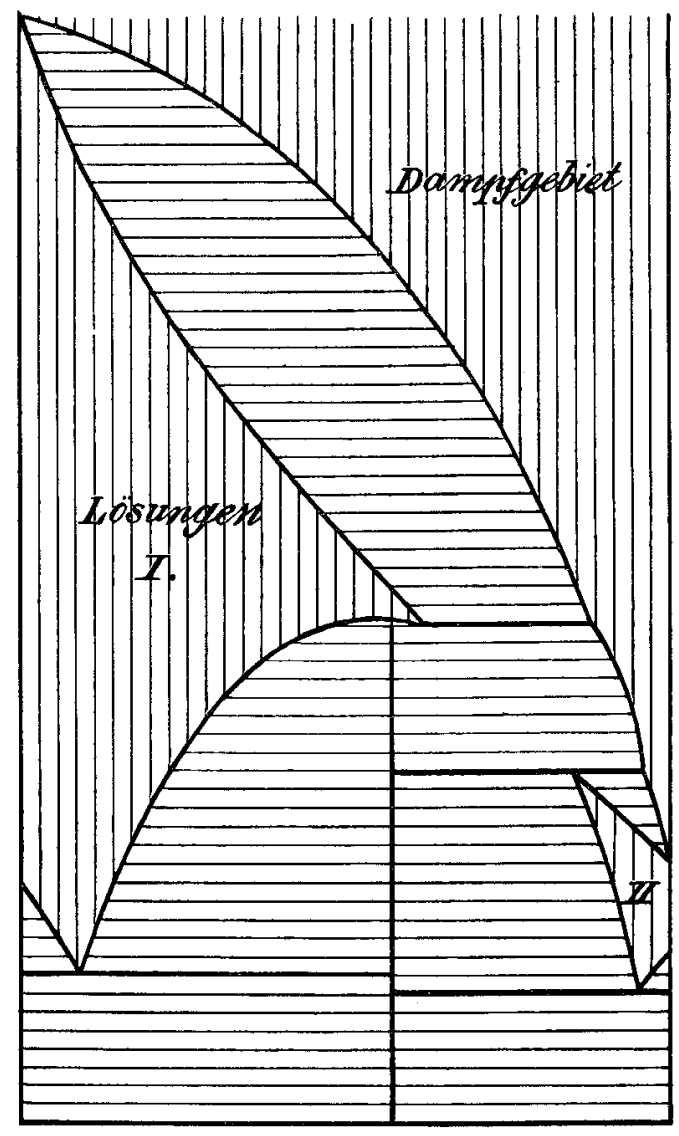

Fig. 9e.

Stücke der Siedekurven, die beiden ersteren für die Flüssigkeiten, die beiden letzteren für die Dämpfe.

$H E, K F$ sind die Löslichkeitskurven von $\mathrm{Bi}_{2} \mathrm{~S}_{\mathbf{s}}$.

$B E$ diejenige für $\mathrm{Bi}, D F$ diejenige für $\mathrm{S}$.

$I L$ gibt die Dämpfe an, welche mit festem $\mathrm{Bi}_{2} \mathrm{~S}_{3}$ koëxistieren.

Es sind also folgende Gebiete zu unterscheiden:

Oberhalb $A I L C$ homogene Dämpfe.

$A H E B$ und $K C D F$ homogene Flüssigkeiten. 
$A H J$ und $K L C$, Flüssigkeit neben Dampf.

$H M O E, K F P N$, Lösung neben festem $\mathrm{Bi}_{2} \mathrm{~S}_{3}$.

$B E Q$, Lösung neben festem Bi.

$D R F$, Lösung neben festem $\mathrm{S}$.

$M J L N$, Dampf neben festem $\mathrm{Bi}_{2} \mathrm{~S}_{3}$.

Unterhalb $Q E O$, festes $\mathrm{Bi}$ neben festem $\mathrm{Bi}_{2} \mathrm{~S}_{3}$.

Unterhalb $P F R$, festes $\mathrm{Bi}_{2} \mathrm{~S}_{3}$ neben festem $\mathrm{S}$.

Es erhebt sich, was die Bedeutung der Figur anbetrifft, jedoch noch die Frage, ob die Lösungen, deren Gebiete durch I und II angegeben sind, Teile einer in allen Verhältnissen mischbaren Serie sind oder nicht.

Theoretisch lärst sich hierüber kein Aufschlufs bekommen, denn eine ganz ähnliche Figur wie $9 \mathrm{~d}$ gilt (nach noch nicht publizierten Betrachtungen von Prof. BakHuIs RoozeBoom) auch für letzteren Fall. Die ganze Figur ist z. B. auf das System $\mathrm{H}_{2} \mathrm{O}+\mathrm{SO}_{2}$ anwendbar, wenn $M$ das Hydrat darstellt, dessen gesättigte Lösung $H$ bei $7^{0}$ siedet. Da sich aber das Hydrat unterhalb dieser Temperatur nicht sofort aus der Lösung abscheidet, ist es möglich zu zeigen, dafs daselbst zweierlei Lösungen nebeneinander bestehen können. Beim Wismutsulfid ist das nicht möglich, weil es zu rasch kristallisiert.

Es besteht aber noch ein anderes Mittel zur Unterscheidung. Sind nämlich die lösungen I und II mischbar, so soll bei fortgesetzter Erhitzung unter steigendem Druck das Sulfid in eine homogene Flüssigkeit übergehen. Beim Schwefligsäurehydrat ist das nicht der Fall, sondern man sieht dann zwei flüssige Schichten auftreten. Wegen der sehr geringen Löslichkeit des Sulfids in flüssigem Schwefel bei $440^{01}$ erwarte ich, dals das Wismutsulfid dieselbe Erscheinung bieten wird, sobald der Versuch gelingt, demnach die Lösungen I und II nicht zu einer kontinuierlichen Reihe gehören. ${ }^{2}$

Die Figur $9 \mathrm{~d}$ wollen wir schliefslich benutzen, um daraus das

${ }^{1}$ Die Löslichkeit ist so gering, dafs keine Siedepunktserhöhung des Schwefels beobachtet werden konnte. $L$ fällt demnach praktisch mit $C=$ $440^{\circ}$ zusammen und $F K$ mit der Achse $R C$.

2 Bei $\mathrm{Sb}_{2} \mathrm{~S}_{3}$ ist die Nichtmischbarkeit noch stärker prononziert. Nach PécaboN lösen $\mathrm{Sb}_{2} \mathrm{~S}_{3}$ und $\mathrm{Sb}$ einander nur in sehr beschränkter Menge und da überdies $\mathrm{Sb}_{2} \mathrm{~S}_{3}$ auch im flüssigen Schwefel sich sehr wenig löst, so gibt es $z$ wischen $\mathrm{Sb}$ und $\mathrm{S}$ wohl drei Reihen Lösungen. Ein dergleiches Beispiel ist ton mir vor kurzem ausführlich untersucht beim System Pyridin-Jodmethyl. Zeitschr. phys. Chem. $\mathbf{5 3}$ (1905). 
Verhalten einzelner Mischungen beim Erhitzen abzuleiten. Es sind besonders die Mischungen, deren Zusammensetzungen zwischen $H$ und $M$ und zwischen $M$ und $I$ liegen.

1. Die Zusammensetzung der Mischung liegt zwischen $H$ und

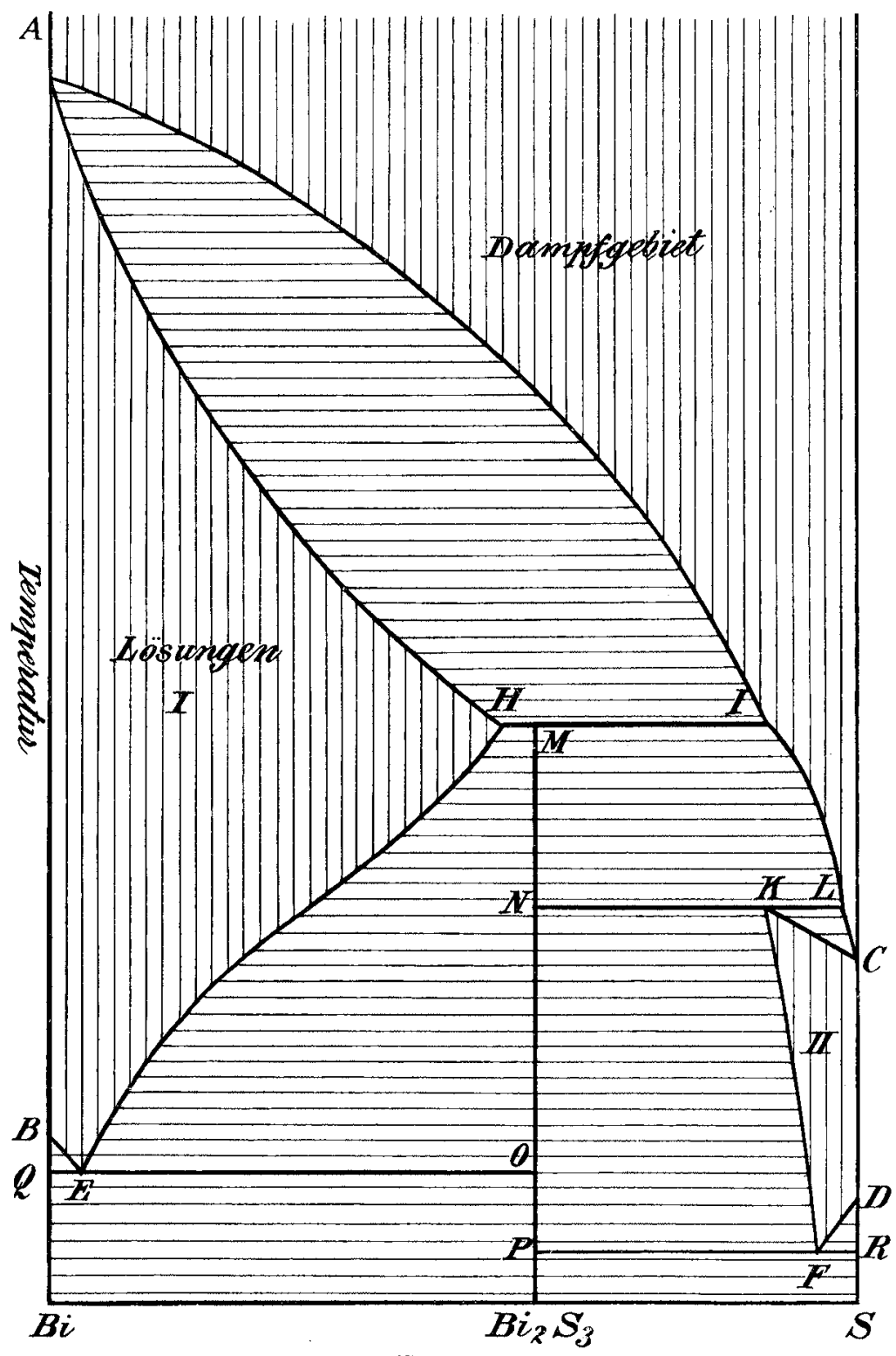

Fig. $9 \mathrm{~d}$. 
M. Bei niedriger Temperatur ist dieselbe ein Gemenge von Bi und $\mathrm{Bi}_{\mathbf{2}} \mathrm{S}_{3}$.

Beim Erhitzen schmilzt zuerst bei der Temperatur von $E$ die eutektische Mischung, bei weiterer Temperaturerhöhung löst sich mehr $\mathrm{Bi}_{2} \mathrm{~S}_{3}$, während die Zusammensetzung der Flüssigkeit der Kurve $E H$ folgt. Dies dauert bis $H$, wo die Flüssigkeit Dampf $J$ bildet, es koëxistieren hier also Flüssigkeit $H$, fester Körper $M$ und Dampf $J$. Man hat hier also eine siedende gesättigte Lösung. Bei Wärmezufuhr findet eine Umwandlung des festen Körpers $M$ in Lösung $H$ und Dampf $J$ statt, d. h. der feste Körper verschwindet.

Unser Fall ist jedoch nicht völlig vergleichbar mit der siedenden gesättigten Lösung in einem binären System, wo die weniger flüchtige Komponente die feste Phase ist, weil dann sich die Lösung in die feste Phase und Dampf zerlegt. Mehr gleicht er dem Fall, dafs die siedende gesättigte Lösung die flüchtigere Komponente als feste Phase enthält, wie bei fester Kohlensäure in Alkohol; ${ }^{1}$ doch ist wiederum ein Unterschied, denn dort bildet sich Dampf aus fester Kohlensäure und Lösung, hier zerfällt der feste Körper in Lösung und Dampf.

2. Wenn die Zusammensetzung zwischen $M$ und $J$ liegt, hat man von der Temperatur des eutektischen Punktes $F$ bis zu der Temperatur von $N$ festes $\mathrm{Bi}_{2} \mathrm{~S}_{3}$ neben Lösung. Bei der Temperatur $N$ spaltet sich die Flüssigkeit in festes $\mathrm{Bi}_{2} \mathrm{~S}_{3}$ und Dampf. Bei höherer Temperatur bleiben dann $\mathrm{Bi}_{2} \mathrm{~S}_{3}$ und Dampf nebeneinander bestehen, bis $M$, wo der feste Körper sich wieder in Flüssigkeit und Dampf spaltet. Man hat hier also mit Mischungen zu tun, welche bei zwei verschiedenen Temperaturen $N$ und $M$ siedende gesättigte Lösungen bilden. Dieser Fall ist aber nicht ganz analog den von BAKHUIS RoOzEBOom ${ }^{2}$ behandelten, denn dort siedet die Lösung bei seinem oberen Siedepunkt bei Abkühlung unter Absetzen des festen Körpers. Der Unterschied liegt darin, dafs dort die Zusammensetzung der Lösung zwischen derjenigen des Dampfes und des festen Körpers liegt, während hier das feste $\mathrm{Bi}_{2} \mathrm{~S}_{3}$ die mittlere Zusammensetzung hat. ${ }^{3}$

1 Bakguis Roozeboom, 1. c. S. 340.

${ }^{2}$ I. c. S. 349 .

${ }^{3}$ Prof. B. R. hat, seit ich dieses schrieb, eine allgemeine Betrachtung über die Siedepunkte gesättigter Lösungen in der Oktobersitzung der Kön. Akad. zu Amsterdam gegeben. 
Die bekannte Tatsache, dafs natürliches und künstliches $\mathrm{Bi}_{2} \mathrm{~S}_{3}$ beim Erhitzen ein Sublimat von Schwefel gibt, ist nun ohne weiteres verständlich.

Das in Lösung vorhandene $\mathrm{Bi}_{2} \mathrm{~S}_{3}$ mufs offenbar teilweise dissoziiert sein, denn sonst würde die Flüssigkeit $H$ keinen Dampf geben, welcher fast ausschliefslich aus Schwefel bestand, sondern einen Dampf, dessen Zusammensetzung zwischen $\mathrm{Bi}_{2} \mathrm{~S}_{3}$ und Bi lag. Wegen des grofsen Unterschiedes zwischen den Siedepunkten des Schwefels und denjenigen von $\mathrm{Bi}_{2} \mathrm{~S}_{3}$ und $\mathrm{Bi}$ kann aber die Dissoziation sehr gering sein und dennoch der Dampf fast nur Schwefel enthalten.

Die oben geschilderte Sachlage wird man öfters antreffen, wenn der Schmelzpunkt einer Verbindung sehr weit oberhalb des Siedepunktes einer ihrer Komponenten liegt. Bei Zinn und Phosphor z. B. treten dieselben Erscheinungen auf. Wenn man in geschmolzenem Zinn roten Phosphor einträgt, so verbinden beide sich, die anfangs dünnflüssige Masse wird breiartig durch Kristalle von $\mathrm{Sn}_{3} \mathrm{P}$. Versucht man aber im offenen Rohre diese Kristalle zu schmelzen, so fängt die Flüssigkeit zu sieden an, während noch festes $\mathrm{Sn}_{3} \mathrm{P}$ anwesend ist, und bei fortwährendem Sieden verschwindet der feste Körper ganz, und Phosphordampf entweicht. Es werden sich dergleiche Verhältnisse bei hochschmelzenden ziemlich stabilen Verbindungen noch wohl vielfach auffinden lassen.

Zum Schlufs noch eine Bemerkung über die Bedeutung der jetzigen Untersuchung für die Interpretierung einer anderen Versuchsreihe von Pelabon. Err hatte schon früher ${ }^{1}$ eine Untersuchung über das Gleichgewicht $z$ wischen Schwefelwasserstoff, Wasserstoff, Wismut und Wismutsulfid angestellt und gelangte dabei zu Resultaten, welche ganz mit denjenigen der Schmelzkurve übereinstimmen. Man hat hier mit einem Systeme dreier Komponenten H, S und $\mathrm{Bi}$ zu tun, folglich ist das System bei konstanter Temperatur und konstantem Drucke nonvariant wenn drei Phasen, univariant wenn zwei Phasen anwesend sind. Wenn man also Schwefelwismutmischungen von verschiedener Zusammensetzung neben $\mathrm{H}_{2}$ und $\mathrm{H}_{2} \mathrm{~S}$ bei einer Temperatur untersucht, wo sie alle flüssig sind, so sind nur zwei Phasen anwesend, Dampf und Flüssigkeit; das System besitzt dann eine Freiheit, das heilst die Zusammensetzung des Dampfes, der Schwefelwasserstoffmischung, ändert sich mit dem Mischungsverhältnisse von Schwefel und Wismut. Wenn aber die

${ }^{1}$ Ann. chim. phys. VII, 25 (1902), 424. 
Temperatur eine solche ist, dafs die Mischungen nicht homogen flüssig sind, sondern noch eine feste Phase anwesend ist, so ist das System nonvariant, d. h. die Zusammensetzung des Gases ist bei konstanter Temperatur und Druck unabhängig von dem Mischungsverhältnisse von $B i$ und $S$ innerhalb der Grenzen, zwischen denen die Natur der festen Phase dieselbe bleibt.

Bei $440^{\circ}$ enthält die an Sulfid gesättigte Lösung etwa 4 Atomproz. S. Deshalb wird bei grölserem Schwefelgehalt die Zusammensetzung des Gases von demselben unabhängig sein, wie Pḱtabon auch gefunden hat. Weil er aber nicht über $50 \% \mathrm{~S}$ hinausgegangen ist, darf diese Konstanz nicht als Beweis angesehen werden, dals BiS die feste Phase ist. Wenn, wie ich meine, ihre Zusammensetzung $\mathrm{Bi}_{2} \mathrm{~S}_{3}$ ist, so hätte bei Fortsetzung der Untersuchung die Konstanz des Gases bis zu 60 Atomproz. S fortdauern müssen.

Schliefslich danke ich Herrn Prof. B. Roozenoom dafür, dafs er meine Aufmerksamkeit auf die allgemeine Bedeutung lenkte, die eine erneute Untersuchung des jetzt betrachteten Systems haben konnte.

Amsterdam, Chem. Laboratorium der Universität, August 1905.

Bei der Redaktion eingegangen am 6. September 1905. 
Zeitschrift für anorganische Chemie. Band 47.

Taf. 3.
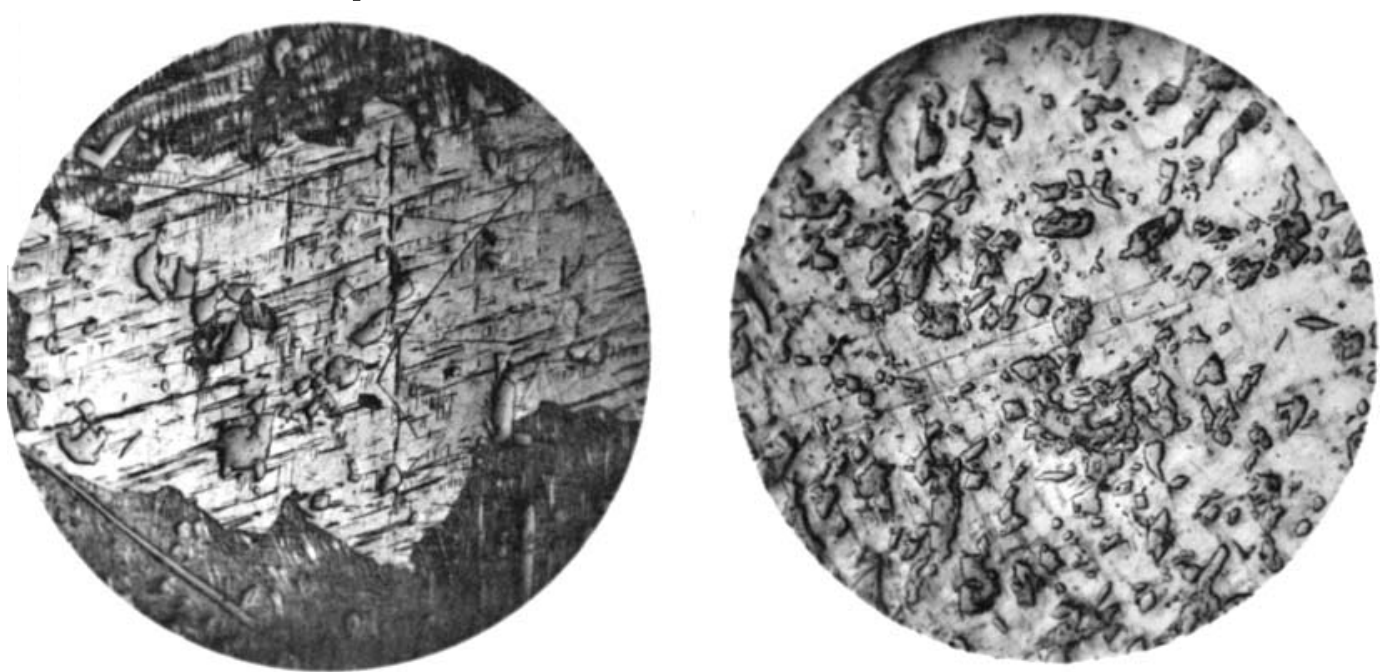

Fig. 3. 4.2 Atompror. S.

Fig. 4. 17,2 Atomproc. S.
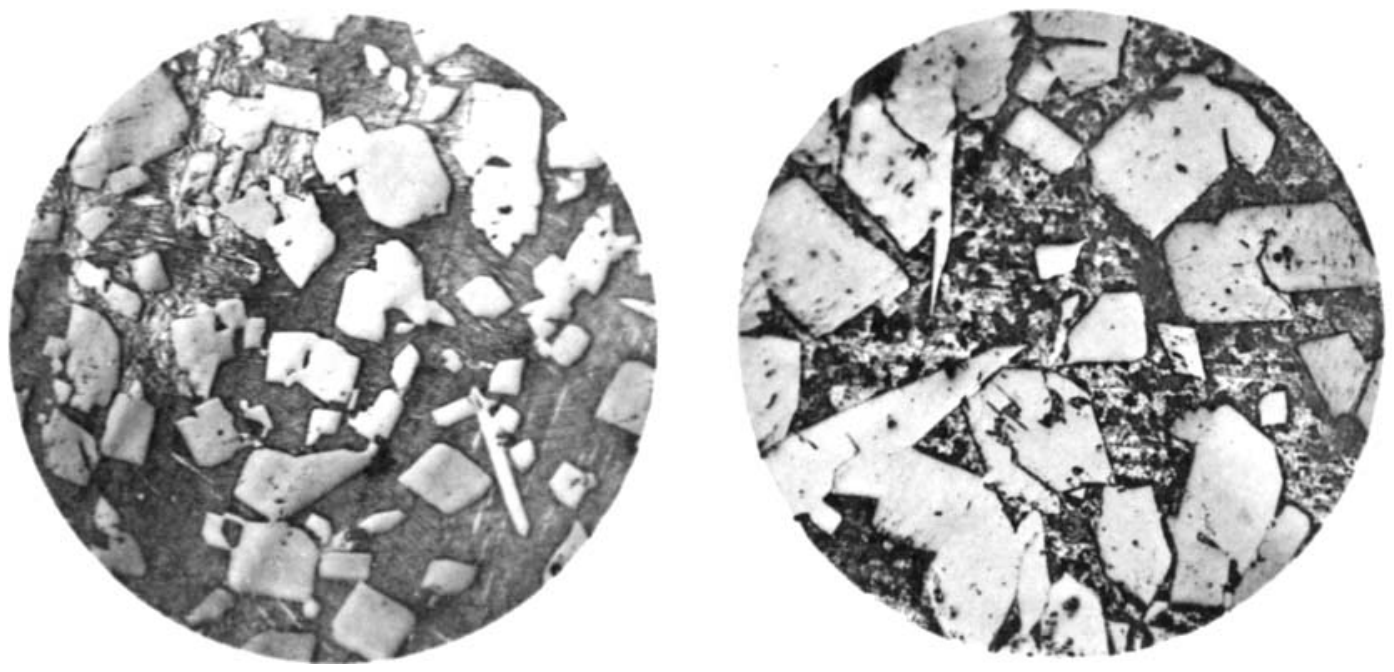

Fig. 5. $28,8 \wedge$ tomproc, $\$$.
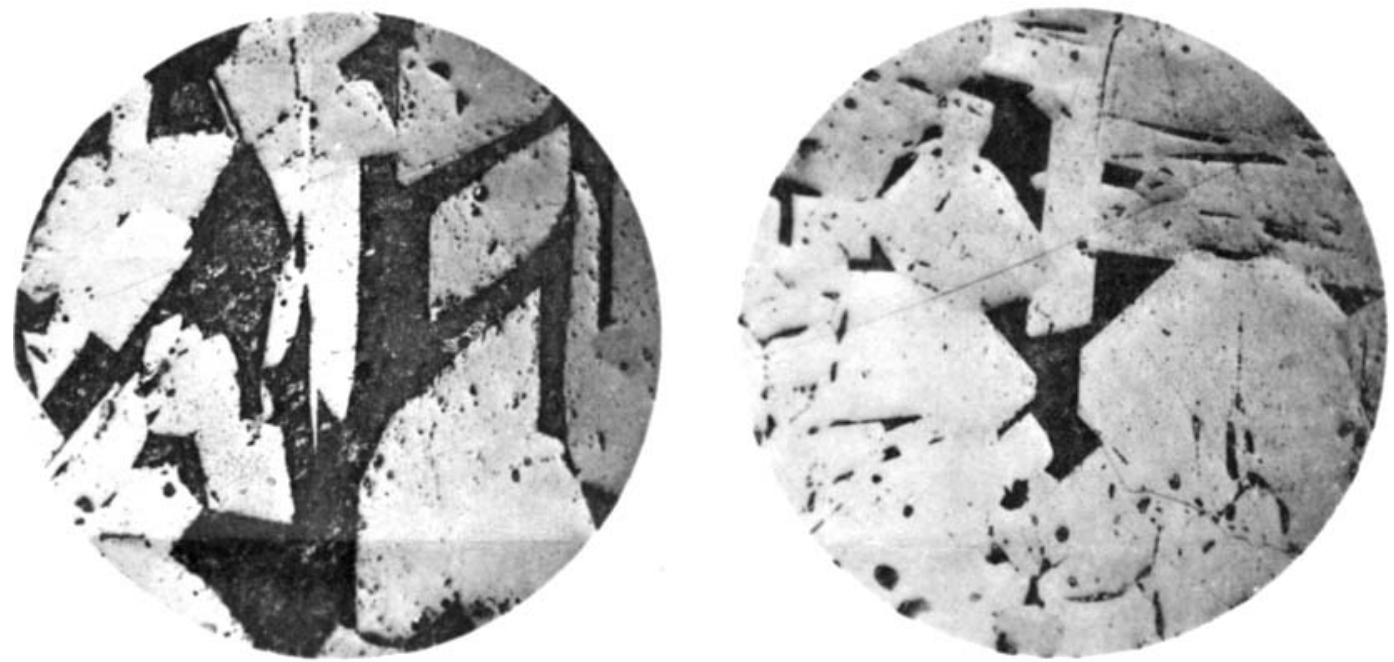

jig. 7, 46,9 .ltomprow..

Fig. 8. 52,4 Atomproc. S. 